\title{
latrogenic cerebrospinal fluid leak after repeated nasal swab tests for COVID-19: illustrative case
}

\author{
Johnson Ku, BS, ${ }^{1}$ Chieh-Yi Chen, MD, ${ }^{2,3}$ Jason Ku, BS, ${ }^{1}$ Hsuan-Kan Chang, MD, ${ }^{2,3,4}$ Jau-Ching Wu, MD, PhD, ${ }^{2,3,5}$ and \\ Yu-Shu Yen, $\mathrm{MD}^{2,3}$
}

\begin{abstract}
${ }^{1}$ Department of Life Sciences, University of California, Los Angeles, California; ${ }^{2}$ Department of Neurosurgery, Neurological Institute, Taipei Veterans General Hospital, Taipei, Taiwan; and ${ }^{3}$ College of Medicine, ${ }^{4}$ Department of Biomedical Imaging and Radiological Sciences, and ${ }^{5}$ Institute of Pharmacology, National Yang Ming Chiao Tung University, Taipei, Taiwan
\end{abstract}

BACKGROUND Nasal swab tests are one of the most essential tools for screening coronavirus disease 2019 (COVID-19). The authors report a rare case of iatrogenic cerebrospinal fluid (CSF) leak from the anterior skull base after repeated nasal swab tests for COVID-19, which was treated with endoscopic endonasal repair.

OBSERVATIONS A 41-year-old man presented with clear continuous rhinorrhea through his left nostril for 5 days after repeated nasal swabbing for COVID-19. There were no obvious risk factors for spontaneous CSF leak. Computed tomography cisternography showed contrast accumulation in the left olfactory fossa and along the left nasal cavity. Such findings aligned with a preliminary diagnosis of CSF leakage through the left cribriform plate. Magnetic resonance imaging confirmed the presence of a CSF fistula between his left cribriform plate and superior nasal concha. The patient underwent endoscopic endonasal repair. CSF rhinorrhea ceased after the surgery, and no recurrence was noted during the 12-week postoperative follow-up period.

LESSONS Although rare, iatrogenic CSF leakage can be a serious complication following COVID-19 nasal swab tests, especially when infection may cause significant neurological sequelae. Healthcare providers should become familiar with nasal cavity anatomy and be well trained in performing nasal swab tests.

https://thejns.org/doi/abs/10.3171/CASE21421

KEYWORDS COVID-19; iatrogenic CSF leak; CSF fistula; CSF rhinorrhea; nasal swab test

Since its outbreak, coronavirus disease 2019 (COVID-19) has resulted in more than 171 million confirmed cases and caused more than 3.7 million deaths worldwide. ${ }^{1}$ Acute respiratory distress syndrome and respiratory failure develop in many patients with severe COVID-19. Additionally, multiple organ failure, such as acute cardiac, kidney, and liver injury, in addition to cardiac arrhythmias, rhabdomyolysis, coagulopathy, and shock, may result from COVID-19. ${ }^{2}$ Besides acute respiratory syndrome, viral infection may also lead to serious neurological complications, including loss of smell and taste, confusion, encephalitis, and GuillainBarré syndrome. $^{3}$ While the prevalence of neurological symptoms remains undetermined, estimates indicate that between $30 \%$ to $50 \%$ of hospitalized patients with COVID-19 have neurological issues. ${ }^{4}$

Adequate testing methods are essential for containing and treating the pandemic. One of the most widely used screening methods for COVID-19 is the nasal swab test. Since February 2020, more than 380 million tests have been performed in United States, with a million more administered daily. ${ }^{5}$ However, the safety of nasal swab tests is usually ignored, and reported complications in literature are scarce. Here, we present a case of iatrogenic cerebrospinal fluid (CSF) leak after repeated nasal swab test for COVID-19 who required endoscopic endonasal repair.

\section{Illustrative Case}

A 41-year-old man and frequent international traveler presented himself to the hospital. He had previously undergone multiple nasal swab tests during 2020, with his latest accounts describing insertion via his left nostril by a physician. The test was described as particularly painful, but no nasal bleeding was noted afterwards. Clear rhinorrhea

ABBREVIATIONS COVID-19 = coronavirus disease 2019; CSF = cerebrospinal fluid; $C T$ = computed tomography; $\mathrm{MRI}=$ magnetic resonance imaging . INCLUDE WHEN CITING Published October 25, 2021; DOI: 10.3171/CASE21421.

SUBMITTED July 21, 2021. ACCEPTED September 9, 2021.

(c) 2021 The authors, CC BY-NC-ND 4.0 (http://creativecommons.org/licenses/by-nc-nd/4.0/). 


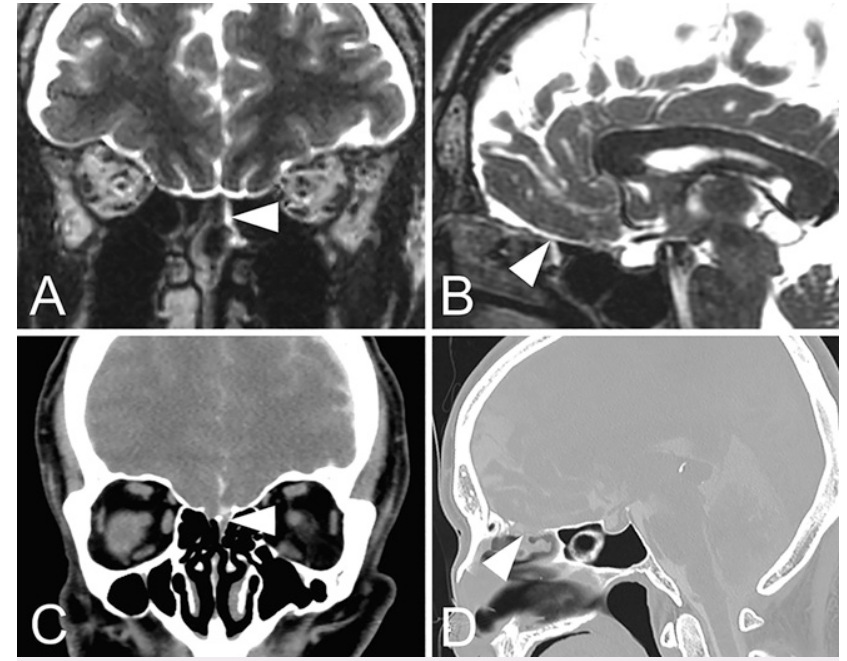

FIG. 1. A and B: MRI showed T2-weighted hyperintensity signal (arrowheads) over the left cribriform plate to the left superior nasal concha, indicating CSF fistula. C and D: CT cisternography showed contrast accumulation (arrowheads) over the left superior nasal cavity on 4-hour post contrast image.

through the patient's left nostril while leaning forward (Video 1) was noted 5 days after the last nasal swab test. A subsequent beta-2-transferrin test returned positive and CSF rhinorrhea was confirmed at the local hospital. The patient had no history of idiopathic intracranial hypertension, endonasal surgery, or obstructive sleep apnea, and he was not overweighted. Magnetic resonance imaging (MRI) showed a T2-weighted hyperintensity signal (Fig. $1 \mathrm{~A}$ and $\mathrm{B}$ ) over the left cribriform plate extending to the left superior nasal concha. Computer tomography (CT) cisternography (Fig. $1 \mathrm{C}$ and D) identified contrast accumulation in the left olfactory fossa and along the left nasal cavity. These findings remain consistent with CSF leakage through the left cribriform plate. The patient subsequently underwent endoscopic endonasal repair (Video 2) after his COVID-19 tests proved negative. Persistent CSF leakage was observed at the medial side of the left superior nasal concha base (Fig. 2A), and the leakage site was confirmed via intraoperative navigation (Fig. 2B). After denuding the surrounding mucosa, an autologous fascia graft harvested from the anterior sheath of the rectus abdominis muscle was used, and an onlay method was adopted to cover the fistula (Fig. 2C). The graft was sealed with a fibrin sealant and packed with an autologous fat graft. The repair layers were supported postoperatively for 3 days with a 14 French Foley catheter placed within the left nostril. The patient also underwent continuous lumbar drainage for 5 days postoperatively. The CSF rhinorrhea ceased after the repair surgery, and there was no recurrence noted at the 12-week postoperative follow-up.

VIDEO 1. Clip showing clear rhinorrhea through left nostril while the patient is leaning forward. Click here to view.

VIDEO 2. Clip showing endoscopic endonasal repair of the CSF leak. Persistent CSF leakage was observed at the medial side of the left superior nasal concha base and was confirmed with intraoperative navigation system. After denuding the surrounding mucosa, an autologous fascia graft harvested from the anterior sheath of the rectus abdominis muscle was used with an onlay method to cover the fistula. The graft was sealed with a fibrin sealant and packed with an autologous fat graft. The repair layers were supported with a 14 French Foley catheter inflated within the left nostril. Click here to view.

\section{Discussion \\ Observations}

CSF leakage is a serious medical condition and can be initially mistaken for allergic rhinitis. If left untreated, this can lead to serious central nervous system infection. CSF leak may result from traumatic, nontraumatic, and iatrogenic causes, while female gender, obesity, intracranial hypertension, and obstructive sleep apnea are risk factors for spontaneous CSF leakages. ${ }^{6}$ Thin-cut CT scans are used for detecting bony defects, while MRIs may help in the screening of encephalocele, hydrocephalus, or other pathologies. CT cisternography is used if no definite defect can be found based on CT alone. In our case, endoscopic repair was performed. Due to only a small bony defect without encephalocele, we opted to preserve the superior and middle nasal concha and utilized an autologous fascia and fat graft to cover the defect with an onlay and multilayer method.

Nasal swab tests that sample upper respiratory secretion via the nasopharynx is one of the main methods recommended by the Centers for Disease Control and Prevention for screening COVID$19 .^{7}$ Although adverse events related to nasal swab tests are usually rare and minor, ${ }^{8}$ three cases of iatrogenic CSF leak after nasal swab tests were reported, and two of them required surgical repair (Table 1). ${ }^{9-11}$ Sullivan et al. reported the first case of iatrogenic CSF leak after a nasal swab test for COVID-19. ${ }^{9}$ The patient had a
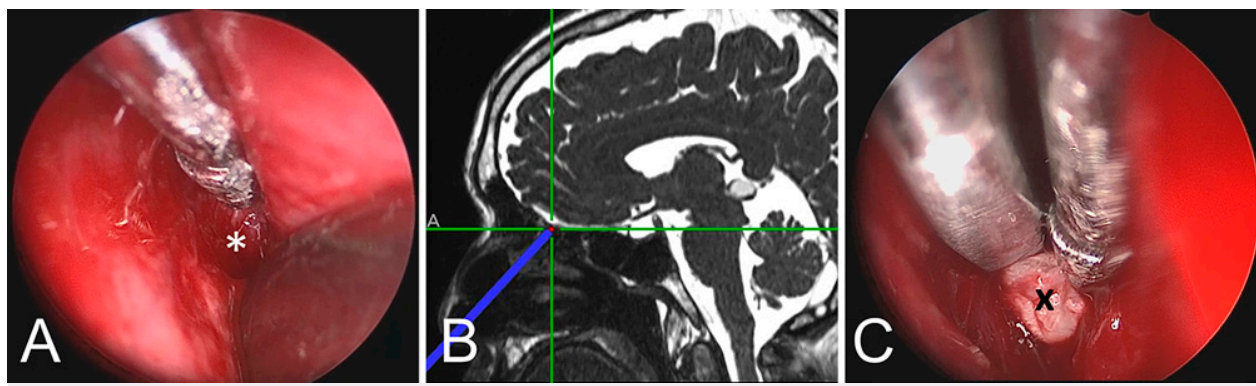

FIG. 2. A: Endoscopy view of CSF leakage site (asterisk) noted medial to the root of the left superior nasal concha. B: The position of fistula was confirmed with intraoperative navigation. C: Autologous fascia graft ("X" mark) harvest from anterior rectus sheath was used with an onlay method for covering the fistula. 
TABLE 1. Comparison of iatrogenic CSF leak cases after nasal swab test for COVID-19

\begin{tabular}{|c|c|c|c|c|c|c|c|}
\hline Authors \& Year & $\begin{array}{c}\text { Age (yrs), } \\
\text { Sex }\end{array}$ & Risk Factor & Location & Bone Defect & Encephalocele & Meningitis & Surgery \\
\hline Present case & $41, M$ & None & Left cribriform plate & - & - & - & + \\
\hline $\begin{array}{l}\text { Alberola-Amores } \\
\text { et al., } 2021^{10}\end{array}$ & $41, F$ & Not mentioned & Left cribriform plate & Not mentioned & - & + & - \\
\hline
\end{tabular}

"+" means that the above condition was found in the case. "-" means that the above condition was not found in the case.

history of idiopathic intracranial hypertension and a previous surgery involving the removal of nasal polyp with a skull base bony defect at the anterior fossa and a large encephalocele. The authors theorize that the swab violated the patient's preexisting encephalocele and caused the subsequent CSF leak. Alberola-Amores et al. reported another case of CSF leak 1 week after the nasal swab test, which later developed into meningitis. ${ }^{10}$ The fistula was found at the left cribriform plate, and he received medical treatments only. The CSF leak resolved spontaneously, and the authors inferred that the inflammation of the meninges formed granulation tissue that may have closed the fistula. Paquin et al. reported the third case without previous surgery or trauma history. ${ }^{11}$ The CT scan showed a bony defect in the posterior right cribriform plate, and the MRI showed an encephalocele projecting from the olfactory fossa of the right superior nasal cavity. The patient then underwent endonasal repair surgery and recovered well.

Including our case, the leakage sites of all four cases were found over the anterior skull base near the midline. In our opinion, iatrogenic CSF leakages after a nasal swab test may result from unfamiliarity with the

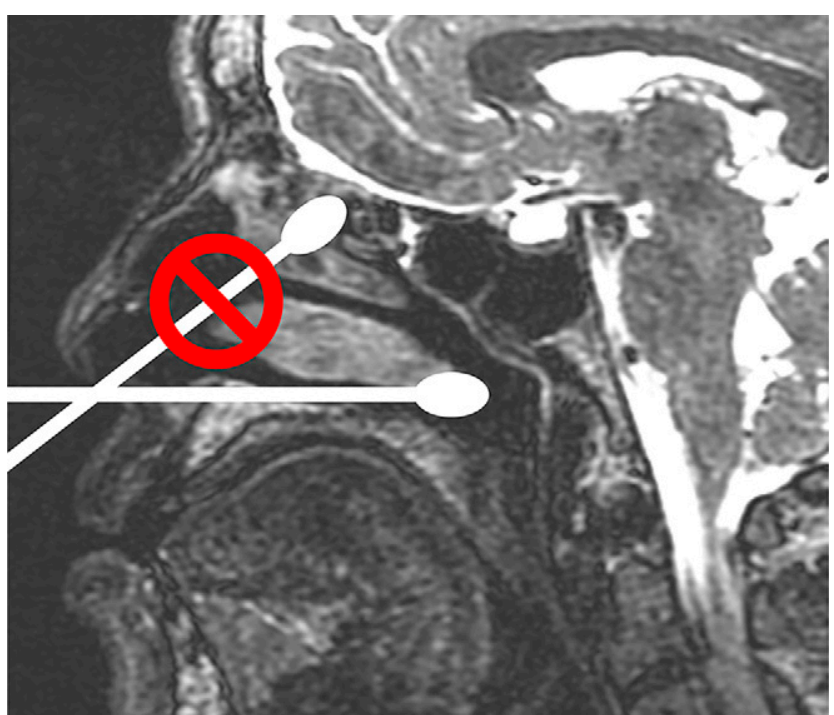

FIG. 3. Illustration showing the different trajectories while performing nasal swab tests. The correct trajectory should be parallel to the palate aiming for nasopharynx. The incorrect diagonal trajectory along the external naris may lead to frontal skull base or cribriform plate damage. anatomy of nasal cavity. For obtaining a nasopharynx specimen, the healthcare provider should insert the swab into the nostril, parallel to the palate, close to the floor of nasal canal. ${ }^{12}$ In a nationwide Canadian study, Hiebert et al. found some heterogeneity in guidance for healthcare providers regarding nasopharyngeal swab performance. ${ }^{13}$ Trauma to the skull base can be caused by inserting the swab diagonally upwards along the shape of nose and external naris (Fig. 3). A nasal swab test should be performed with caution, especially in patients with potential COVID-19 infection, in whom neurological syndromes are common and often quite severe. It is intuitive that an iatrogenic CSF leak may induce direct invasion of viral infection to the central nerve system. ${ }^{14}$ As a result, subsequent treatment for CSF leakages can be complicated in a confirmed case of COVID-19.

\section{Lessons}

Although rare, CSF leak can be a seriously adverse event that could occur after nasal swab tests. It is essential that healthcare providers are familiar with anatomy and well trained in performing nasal swabs to avoid further complications.

\section{References}

1. World Health Organization. WHO Coronavirus Disease (COVID-19) Dashboard. Accessed June 1, 2021. https://covid19.who.int/table

2. Berlin DA, Gulick RM, Martinez FJ. Severe Covid-19. N Engl J Med. 2020;383(25):2451-2460.

3. Paterson RW, Brown RL, Benjamin L, et al. The emerging spectrum of COVID-19 neurology: clinical, radiological and laboratory findings. Brain. 2020;143(10):3104-3120.

4. Fotuhi M, Mian A, Meysami S, Raji CA. Neurobiology of COVID-19. J Alzheimers Dis. 2020;76(1):3-19.

5. Global Change Data Lab. Our world in data Total COVID-19 tests. Accessed June 1, 2021. https://ourworldindata.org/grapher/full-listtotal-tests-for-covid-19?time $=2020-02-20$.latest

6. Lobo BC, Baumanis MM, Nelson RF. Surgical repair of spontaneous cerebrospinal fluid (CSF) leaks: a systematic review. Laryngoscope Investig Otolaryngol. 2017;2(5):215-224.

7. Centers for Disease Control and Prevention. Interim Guidelines for Collecting, Handling, and Testing Clinical Specimens for COVID-19. Accessed June 1, 2021. https://www.cdc.gov/coronavirus/2019-ncov/ lab/guidelines-clinical-specimens.html\#specimen

8. Föh B, Borsche M, Balck A, et al. Complications of nasal and pharyngeal swabs: a relevant challenge of the COVID-19 pandemic? Eur Respir J. 2021;57(4):2004004.

9. Sullivan CB, Schwalje AT, Jensen M, et al. Cerebrospinal fluid leak after nasal swab testing for coronavirus disease 2019. JAMA Otolaryngol Head Neck Surg. 2020;146(12):1179-1181. 
10. Alberola-Amores FJ, Valdeolivas-Urbelz E, Torregrosa-Ortiz M, Alvarez-Sauco M, Alom-Poveda J. Meningitis due to cerebrospinal fluid leak after nasal swab testing for COVID-19. Eur J Neurol. Published online January 21, 2021. doi: 10.1111/ene.14736

11. Paquin R, Ryan L, Vale FL, Rutkowski M, Byrd JK. CSF leak after COVID-19 nasopharyngeal swab: a case report. Laryngoscope. Published online February 12, 2021. doi: 10.1002/lary.29462

12. Marty FM, Chen K, Verrill KA. How to obtain a nasopharyngeal swab specimen. N Engl J Med. 2020;382(22):e76.

13. Hiebert NM, Chen BA, Sowerby LJ. Variability in instructions for performance of nasopharyngeal swabs across Canada in the era of COVID-19 - what type of swab is actually being performed? J Otolaryngol Head Neck Surg. 2021;50(1):5.

14. Solomon T. Neurological infection with SARS-CoV-2 - the story so far. Nat Rev Neurol. 2021;17(2):65-66.

\section{Disclosures}

The authors report no conflict of interest concerning the materials or methods used in this study or the findings specified in this paper.

\section{Author Contributions}

Conception and design: Chen, Johnson Ku, Chang, Wu. Acquisition of data: Chen, Johnson Ku, Yen. Analysis and interpretation of data: Chen, Johnson Ku, Jason Ku, Wu. Drafting the article: Chen, Johnson $\mathrm{Ku}$, Jason Ku. Critically revising the article: Johnson Ku, Jason Ku, Chang, Wu. Reviewed submitted version of manuscript: all authors. Approved the final version of the manuscript on behalf of all authors: Chen. Statistical analysis: Johnson Ku. Administrative/technical/material support: Chang, Wu, Yen. Study supervision: Chang, Wu.

\section{Supplemental Information \\ Videos}

Video 1. https://vimeo.com/601875745.

Video 2. https://vimeo.com/601879076.

\section{Correspondence}

Chieh-Yi Chen: Neurological Institute, Taipei Veterans General Hospital, Taipei, Taiwan. blacatae@gmail.com. 